\title{
Estimation of Effective Crosslink Density in Poly( $N$-hydroxyalkyl L-glutamine) Hydrogels
}

\author{
Eiji Nakanishi, Kouichi Hamada, Eigo Sugiyama, \\ Sadao HibI, and Toshio HaYASHI* \\ Department of Materials Science \& Engineering, Nagoya Institute of Technology, \\ Gokiso-cho, Showa-ku, Nagoya 466, Japan \\ * Research Center for Biomedical Engineering, Kyoto University, \\ 53, Shogoin-Kawahara-cho, Sakyo-ku, Kyoto 606, Japan
}

(Received October 3, 1990)

\begin{abstract}
Four kinds of poly( $N$-hydroxyalkyl L-glutamine) hydrogels, PHEG, PHPG, PHBG and PHPeG, were prepared by aminolysis of poly $(\gamma$-benzyl L-glutamate) (PBLG) membranes with corresponding aminoalcohols, such as 2-amino-1-ethanol (E), 3-amino-1-propanol (P), 4-amino-1-butanol (B), and 5-amino-1-pentanol (Pe), together with a crosslinking agent octamethylenediamine (OMDA). Effective crosslink density was estimated on the basis of rubber elasticity theory. This estimation was limited to hydrogels which contain random coil polypeptides. The effective crosslink density was shown to be proportional to the ratio of OMDA to amino alcohol. It was found that relative reactivities of OMDA with PBLG are dependent on the alkyl chain lengths of the coexisting aminoalcohols. The order of effective crosslink density was PHBG $>$ PHPG $>$ PHEG, when the same content of OMDA was used.

KEY WORDS Poly( $N$-hydroxyalkyl L-glutamine) Hydrogel / Alkyl Side Chain Length / Rubber Elasticity Theory / Effective Crosslink Density / The Degree of Swelling / Molecular Conformation / Mechanical Property /
\end{abstract}

Hydrogels are water-swollen materials and are usually crosslinked covalently. Mechanical properties of hydrogels are dependent on the degree of swelling and crosslink density. However, it is difficult to know the true crosslink density, even if one can estimate the concentration of the crosslinking agent in polymer, because not all of the crosslinking agents participate in the network formation. Crosslinked poly( $N$-hydroxyalkyl L-glutamine) (PHG) hydrogels are prepared by the competitive substitution of $\gamma$-benzyl L-glutamate $(\gamma$ BLG) residues with amino alcohol and with crosslinking agent in PBLG membranes. ${ }^{1}$ Membrane properties, such as the degree of swelling $Q_{\mathrm{w}}$, molecular conformation, mechanical property, water vapor permeability and enzymatic degradation were investigated for four kinds of PHG hydrogels prepared as different concentration of crosslinking agent in the reaction mixture. ${ }^{2-4}$ However, it is doubtful whether the relative reactivity of 1,8-octamethylenediamine (OMDA) to aminoalcohol depends on the length of aminoalcohols. The effective crosslink density has been estimated extensively on the basis of the rubber elasticity theory ${ }^{5}$ from elastic constants of hydrogels for vinyl polymers ${ }^{6-8}$ and polysaccharides. ${ }^{9}$ In the case of poly(amino acid) hydrogels, it is necessary to take into consideration the effect of molecular conformation which varies with alkyl side chain length. Conformation also affects mechanical strength of hydrogels. ${ }^{2}$ In the present work, four kinds of $\operatorname{poly}(\mathrm{N}$-hydroxyalkyl L-glutamine) hydrogels, PHEG, PHPG, PHBG, and PHPeG, were prepared with various molar concentration of the crosslinking agent ranging $1-5 \mathrm{~mol} \%$ in 
the reaction mixture. The molecular conformation, the degree of swelling and tensile property of the obtained hydrogels were investigated. The object of this study is to estimate the effective crosslink density in poly(amino acid) hydrogels from the rubber elasticity theory by considering the effect of molecular conformation.

\section{EXPERIMENTAL}

\section{Materials}

Synthesis of Mother Polymer. Poly $(\gamma$-benzyl L-glutamate) (PBLG) was synthesized by the $N$-carboxyanhydride (NCA) method. ${ }^{10}$ Trichloromethyl-chloroformate (phosgene dimer) was supplied by Hodogaya Chemical Co. The BLG-NCA was purified by recrystallization from ethyl acetate solution with addition of petroleum ether. Recrystallization was repeated more than three times. Polymerization was initiated with triethylamine (TEA) at an NCA-to-TEA molar ratio of 50 in $1: 1(\mathrm{v} / \mathrm{v})$ mixture of dioxane and dichloromethane. The starting polymer was purified and fractionated as described in a previous paper. ${ }^{11}$ The molecular weight of PBLG was determined $^{12}$ as 348,000 by viscosity measurement in dichloroacetic acid (DCA). All solvents used were distilled twice.

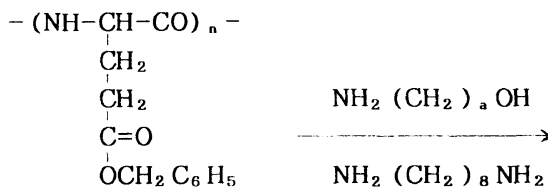

PBLG Membrane
Preparation of Poly(N-hydroxyalkyl L-glutamine) Membranes. Four kinds of $\operatorname{poly}(N$ hydroxyalkyl L-glutamine) (PHG) membranes, PHEG, PHPG, PHBG, and PHPeG, were prepared by the aminolysis of PBLG membranes cast from chloroform solution. PBLG membranes of $c a$. 60 and $1 \mu \mathrm{m}$ in thickness were immersed in the corresponding aminoalcohols, 2-amino-1-ethanol (E), 3-amino-1propanol (P), 4-amino-1-butanol (B), and 5-amino-1-pentanol $(\mathrm{Pe})$, containing 1 to 5 mol $\%$ of OMDA as a crosslinking agent at $58 \pm 0.2^{\circ} \mathrm{C}$. The aminolytic reaction was completed after 7 to 14 days. Debenzylation of $\gamma$-BLG was confirmed by the disappearance of absorption at $250-260 \mathrm{~nm}$ in UV spectra and absorption at 700 and at $750 \mathrm{~cm}^{-1}$ in IR spectra. The absence of $\gamma$-benzyl ester group was confirmed by the disappearance of absorption due to ester groups at $1730 \mathrm{~cm}^{-1}$ in IR spectra. Figure 1 denotes a schematic diagram of the preparation of PHG membranes. The products were washed with distilled water and ethanol exhaustively, and stored in ethanol.

\section{Measurements}

Molecular Conformation of Hydrogels. Molecular conformation of PHG hydrogels was examined by IR (Hitachi Model 285 Infrared

$\begin{array}{cc}-(\mathrm{NH}-\mathrm{CH}-\mathrm{CO})_{1}-(\mathrm{NH}-\mathrm{CH}-\mathrm{CO})_{\mathrm{m}}- \\ \mathrm{CH}_{2} & \mathrm{CH}_{2} \\ 1 & 1 \\ \mathrm{CH}_{2} & \mathrm{CH}_{2} \\ \mathrm{C}=\mathrm{O} & \mathrm{C}=\mathrm{O} \\ 1 & 1 \\ \mathrm{NH}_{1} & \mathrm{NH} \\ \left(\mathrm{CH}_{2}\right)_{\mathrm{a}} & \left(\mathrm{CH}_{2}\right)_{8} \\ \mathrm{OH} & \mathrm{NH} \\ & \mathrm{C}=\mathrm{O} \\ & 1 \\ & \mathrm{CH}_{2} \\ & \mathrm{CH}_{2} \\ & -(\mathrm{NH}-\mathrm{CH}-\mathrm{CO})_{\mathrm{m}}-\end{array}$

$$
\begin{aligned}
& a=2 ; \operatorname{poly}(N \text {-hydroxyethyl L-glutamine) (PHEG) } \\
& a=3 ; \operatorname{poly}(N \text {-hydroxypropyl L-glutamine) (PHPG) } \\
& a=4 ; \operatorname{poly}(N \text {-hydroxybutyl L-glutamine) (PHBG) } \\
& a=5 ; \operatorname{poly}(N \text {-hydroxypentyl L-glutamine) (PHPeG) }
\end{aligned}
$$

Figure 1. General scheme of preparation of crosslinked poly( $N$-hydroxyalkyl L-glutamine) membranes. 
Spectrophotometer) and circular dichroism (CD) (Jasco J-40CD/ORD Spectropolarimeter) measurements. The Amide $\mathrm{V}$ band ${ }^{13}$ in IR spectra was used for identification of molecular conformation. The CD spectra of hydrogels were measured by rotating samples around the optical axis to minimize the effect of linear dichroism. Spectra were taken at every 90 degree rotation and the deviation of observed ellipticity $\theta_{\text {obs }}$ (deg) was found to be within $10 \%$ of the average value. The ratio of $\theta_{\text {obs }}$ in water to that measured in methanol is considered to correspond to the helix content $X_{\mathrm{H}}$, since PHG membranes exhibit completely $\alpha$-helix conformation in methanol. ${ }^{16}$

Degree of Swelling of Hydrogels. The degree of swelling $Q_{\mathrm{w}}(\%)$ was determined by equilibrating the membrane in water at $25^{\circ} \mathrm{C}$. $Q_{\mathrm{w}}$ is given by

$$
Q_{\mathrm{w}}=\frac{W_{\mathrm{w}}-W_{\mathrm{D}}}{W_{\mathrm{D}}} \times 100
$$

where $W_{\mathrm{w}}$ and $W_{\mathrm{D}}$ are the weights of hydrogel and xerogel, respectively. The volume fraction of polymer in hydrogel $v_{2}$ was calculated from the following relation

$$
v_{2}=\frac{V_{\mathrm{D}}}{V_{\mathrm{w}}}=\frac{W_{\mathrm{D}} / \rho_{\mathrm{D}}}{W_{\mathrm{w}} / \rho_{\mathrm{W}}}
$$

The density of xerogels $\rho_{\mathrm{D}}$ was determined by the density gradient method in a carbon tetrachloride-toluene system. The density of hydrogels $\rho_{\mathrm{w}}$ was calculated approximately from the weight fractions of water and xerogel.

Tensile Property of Hydrogels. Tensile modulus of hydrogels was measured in water at $25^{\circ} \mathrm{C}$ using a Tensilon UTM-4LH (Orientec Co.) equipped with a $1 \mathrm{~kg}$ loadcell. A wrapping film was used at chuck parts to prevent hydrogels from breaking or from slippage at the chuck. All hydrogels were tested at a strain rate of $40 \%$ per minute. The Young's modulus $E$, tensile strength $\sigma_{\mathrm{B}}$ and strain at break point $\varepsilon_{\mathrm{B}}$ were estimated from the stress-strain curve.

\section{RESULTS AND DISCUSSION}

\section{Molecular Conformation of Hydrogels}

Linear poly( $N$-hydroxyalkyl L-glutamine)s (PHG) are non-ionic water soluble polymers and molecular conformation of these polymers are dependent on alkyl side chain length. ${ }^{14-16}$ The helix content $X_{\mathrm{H}}$ of linear PHEG, PHPG, PHBG and PHPeG in water are $0 \%, 25 \%$, $70 \%$ and $100 \%$, respectively. ${ }^{15}$ The ratios of the observed ellipticity in water to that in methanol $\theta_{\mathrm{obs}}^{\mathrm{W}} / \theta_{\mathrm{obs}}^{\mathrm{M}}$, which correspond to $X_{\mathrm{H}}$ of crosslinked hydrogels, are shown in Figure 2 as a function of the content of crosslinking agent OMDA in the reaction mixture. For PHEG hydrogel, the random coil conformation is kept until the highest range of OMDA concentration, because of its hydrophilic nature. PHPG and PHBG that exist in interrupted helix conformation in water are converted sharply to the $\alpha$-helix conformation at a certain concentration of OMDA in membrane state. This result indicates that the $\alpha$ helix form is stabilized with increasing crosslink density. This can be explained by the restriction of molecular conformation in a hydrogel due to tight crosslinking.

\section{Degree of Swelling of Hydrogels}

The degree of swelling in a solvent is

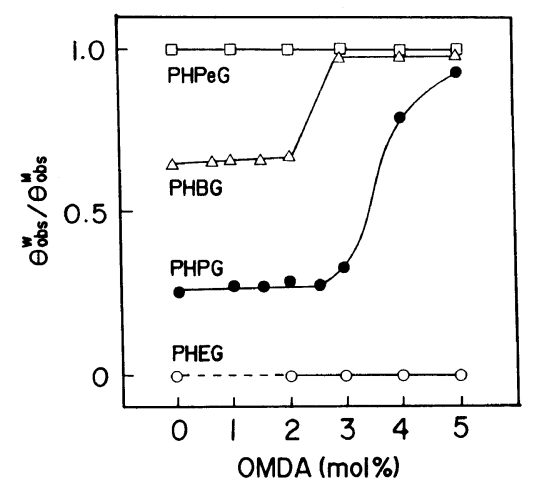

Figure 2. Relationship of the ratio of observed ellipticity in water to that in methanol $\theta_{\mathrm{obs}}^{\mathbf{w}} / \theta_{\mathrm{obs}}^{\mathrm{M}}$ with the molar percent of OMDA in the reaction mixture on poly ( $N$-hydroxyalkyl L-glutamine) membranes in water at $25^{\circ} \mathrm{C}$. 
controlled by the interaction energy between solvent molecules and polymer segments, and this energy is regarded as the elastic energy (crosslink density) for a swollen polymer. When the degree of swelling $Q_{\mathrm{w}}$ is quite large, it is given by the following equation according to the rubber elasticity theory ${ }^{17}$

$$
Q_{\mathrm{W}}{ }^{5 / 3} \fallingdotseq\left(v M_{\mathrm{c}} / V_{1}\right)\left(1-2 M_{\mathrm{c}} / M\right)^{-1}\left(1 / 2-\chi_{1}\right)
$$

where $M_{\mathrm{c}}$ is the molecular weight per crosslinked unit, $M$ is the primary molecular weight, $v$ is the specific volume of polymer, $V_{1}$ is the molar volume of solvent and $\chi_{1}$ is the interaction parameter. The factor $\left(1-2 M_{\mathrm{c}} / M\right)$ expresses the correction for network imperfections resulting from chain ends. It reduces to unity for high molecular weight polymer chains. Equation 1 is simplified to

$$
Q_{\mathrm{W}}{ }^{5 / 3} \fallingdotseq\left(v M_{\mathrm{c}} / V_{1}\right)\left(1 / 2-\chi_{1}\right)
$$

By the effective crosslink density $f_{\mathrm{c}}=K / M_{\mathrm{c}}$, where $K$ is the constant, eq 2 is converted to

$$
\begin{aligned}
\log Q_{\mathrm{w}} \fallingdotseq & -\frac{3}{5} \log f_{\mathrm{c}} \\
& +\frac{3}{5} \log \left[\left(v K / V_{1}\right)\left(1 / 2-\chi_{1}\right)\right]
\end{aligned}
$$

Thus, $\log Q_{\mathrm{w}}$ is in linear relation with $\log f_{\mathrm{c}}$ with the slope as $-3 / 5$.

The effect of OMDA concentration in the reaction mixture on $Q_{\mathrm{w}}$ of the crosslinked hydrogels is shown in Figure 3. $Q_{\mathrm{W}}$ decreases with increasing OMDA molar concentration in the reaction mixture. The slope of the $\log -\log$ plots in PHEG hydrogels which exist in random coil conformation has the value of $-3 / 5$ as predicted by eq 3 . The effective crosslink density is proportional to the OMDA concentration in the reaction mixture. The $\log -\log$ plots of PHPG and of PHBG hydrogels deviate from the linear relationship at the OMDA concentration, at which substantial conformational changes take place as shown in Figure 2. This deviation is considered to be caused by

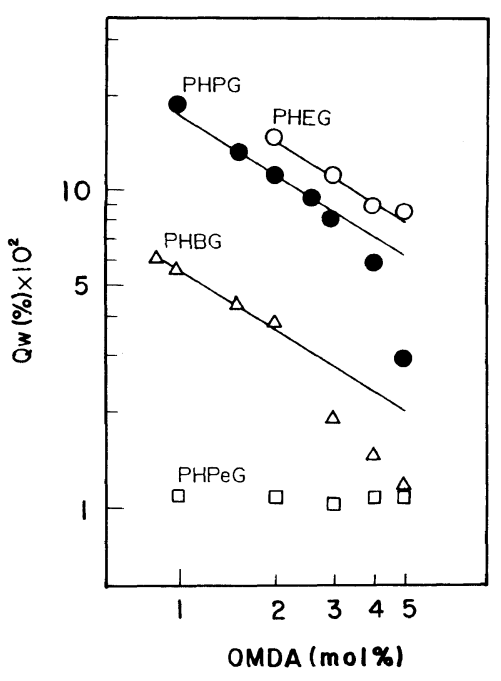

Figure 3. Degree of swelling $Q_{\mathrm{w}}$ as a function of the molar percent of OMDA in the reaction mixture on hydrogels in water at $25^{\circ} \mathrm{C}$. The straight lines indicate the slope of $-3 / 5$.

the decrease of $Q_{\mathrm{w}}$ due to abrupt conformational change from interrupted helix to $\alpha$-helix. PHPeG hydrogel which exists in $\alpha$-helix conformation with low $Q_{\mathrm{w}}$ cannot be dealt with in terms of the rubber elasticity theory. This theory can only be applied to hydrogels which contain random coil region having enough segmental mobility.

\section{Tensile Property of Hydrogels}

Tensile properties of PHG hydrogels are dependent on the alkyl side chain length and on the crosslink density. Figure 4 illustrates the stress--strain (S-S) curves of four kinds of PHG hydrogels with various OMDA concentration in water measured at $25^{\circ} \mathrm{C}$. The numerals in Figure 4 denote the OMDA concentration in the reaction mixture. Table I lists the Young's modulus $E$ at an elongation of $10 \%$, the tensile strength $\sigma_{\mathrm{B}}$ and elongation $\varepsilon_{\mathrm{B}}$ at the break point. Young's modulus is enhanced with increasing OMDA concentration and alkyl side chain length. In natural rubber, it was confirmed that $\varepsilon_{\mathrm{B}}$ decreases with crosslink density and $\sigma_{\mathrm{B}}$ has a maximum value at a low 


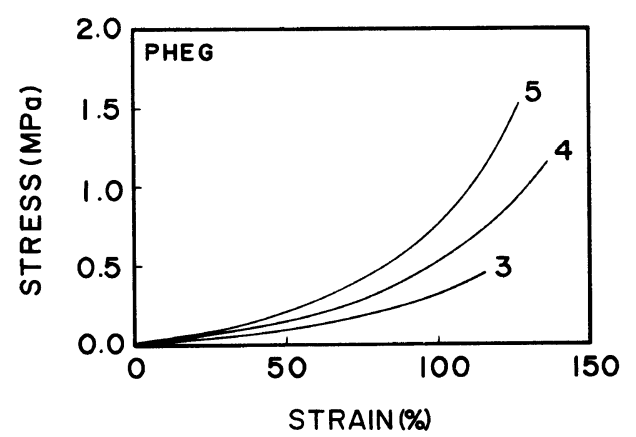

(a)

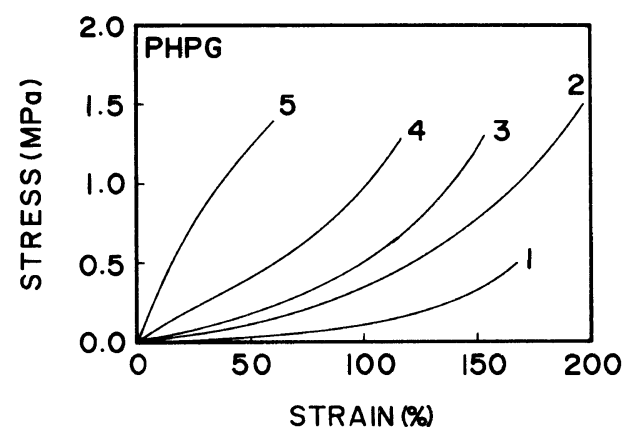

(b)

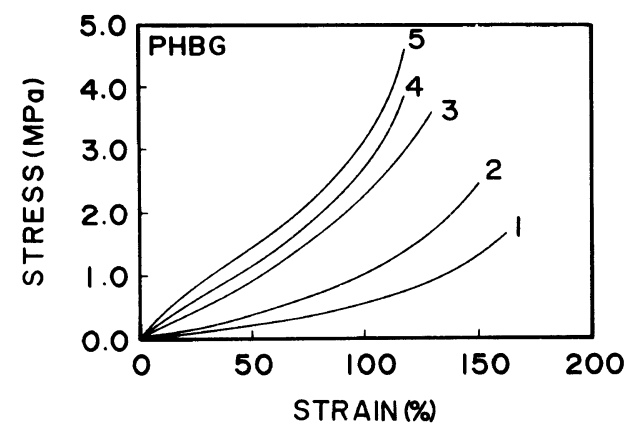

(c)

crosslink density. ${ }^{18}$ Thus $\varepsilon_{\mathrm{B}}$ and $\sigma_{\mathrm{B}}$ in PHG hydrogels have the same trend as natural rubber. Tensile behavior of PHG hydrogels was classified in two types, namely, the skin-type and elastomer-type by the shape of the S-S curve. The skin-type hydrogels show a concave behavior with low Young's modulus as a typical case of human skin, while the elastomer-type hydrogels show a sigmoidal behavior with the transition in the low strain

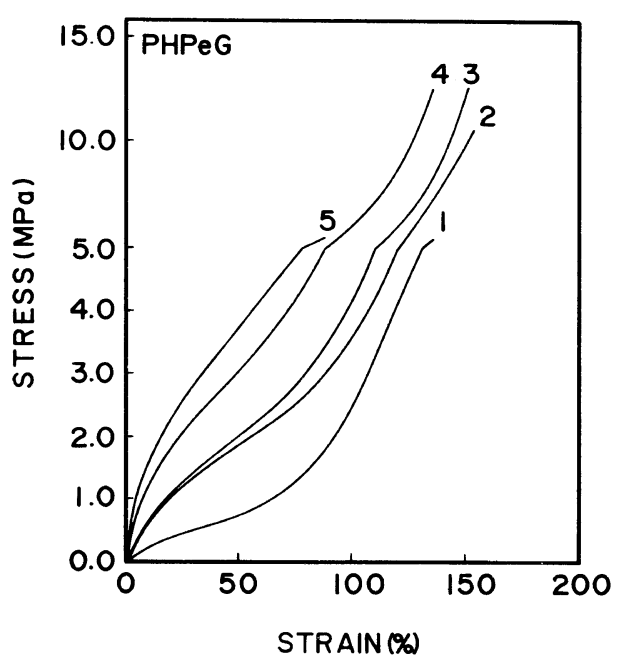

(d)

Figure 4. Stress-strain curves of PHEG (a), PHPG (b), PHBG (c), and PHPeG (d) hydrogels in water at $25^{\circ} \mathrm{C}$. Numerals in the figure denote molar percent of OMDA in the reaction mixture.

region. The skin-type behavior has been accounted for by the entropy elasticity without stress relaxation, creeping and hysteresis, while the elastomer-type behavior has been considered to include the contribution of the energy elasticity with stress relaxation, creeping and hysteresis. The difference in the shape of $\mathrm{S}-\mathrm{S}$ curves between two cases will be discussed in detail elsewhere. ${ }^{19}$ Such PHG hydrogels, which exist in $\alpha$-helix conformation, as PHPeG, PHBG-3, 4, 5, and PHPG-4, 5 show elastomertype behavior, while those which contain random coil conformation such as PHEG, PHPG-1, 2, 3, and PHBG-1, 2 show skin-type behavior. This transition from the skin-type to the elastometer-type occurs at $c a .80 \%$ of helix content, though this transition cannot be provided by the degree of swelling as can be seen in Figure 3. Tensile behavior is considered to depend on the molecular conformation rather than degree of swelling of PHG hydrogels. Moreover, it was shown that eq 3 is applicable to skin-type hydrogels. 
Table I. Mechanical parameters of crosslinked poly $(N$-hydroxyalkyl L-glutamine) hydrogels in water at $25^{\circ} \mathrm{C}$

\begin{tabular}{|c|c|c|c|}
\hline \multirow{2}{*}{$\begin{array}{l}\text { Sample } \\
\text { code }\end{array}$} & $E$ & $\sigma_{\mathrm{B}}$ & $\varepsilon_{\mathrm{B}}$ \\
\hline & $\mathrm{MPa}$ & $\mathrm{MPa}$ & $\%$ \\
\hline PHEG-3 & 0.128 & 0.464 & 115 \\
\hline PHEG-4 & 0.178 & 1.14 & 136 \\
\hline PHEG-5 & 0.221 & 1.52 & 127 \\
\hline PHPG-1 & 0.081 & 0.509 & 167 \\
\hline PHPG-1.5 & 0.130 & 1.05 & 170 \\
\hline PHPG-2 & 0.168 & 1.18 & 196 \\
\hline PHPG-2.5 & 0.216 & 1.18 & 168 \\
\hline PHPG-3 & 0.266 & 1.27 & 151 \\
\hline PHPG-4 & 0.758 & 1.28 & 116 \\
\hline PHPG-5 & 3.29 & 1.39 & 60 \\
\hline PHBG-0.7 & 0.194 & 1.01 & 118 \\
\hline PHBG-1 & 0.246 & 1.61 & 161 \\
\hline PHBG-1.5 & 0.321 & 2.25 & 172 \\
\hline PHBG-2 & 0.393 & 2.47 & 151 \\
\hline PHBG-3 & 1.39 & 3.53 & 126 \\
\hline PHBG-4 & 2.55 & 3.81 & 117 \\
\hline PHBG-5 & 3.92 & 4.52 & 107 \\
\hline PHPeG-1 & 2.64 & 5.30 & 136 \\
\hline PHPeG-2 & 6.16 & 10.7 & 153 \\
\hline PHPeG-3 & 6.53 & 12.7 & 133 \\
\hline PHPeG-4 & 13.3 & 12.5 & 136 \\
\hline PHPeG-5 & 15.5 & 5.49 & 87 \\
\hline
\end{tabular}

\section{Estimation of Effective Crosslink Density of} Hydrogels

The effective crosslink density of elastomer can be estimated by the rubber elasticity theory assuming the Gaussian chain. ${ }^{5}$ The retractive force $\tau$ of a swollen elastomer under network deformation is given by ${ }^{20}$

$$
\begin{gathered}
\tau=G\left(\lambda-1 / \lambda^{2}\right) \\
G=R T v_{e} v_{2}{ }^{1 / 3}
\end{gathered}
$$

where $G$ is the bulk modulus, $\lambda$ is the elongation ratio, $v_{\mathrm{e}}\left(\mathrm{mol} \mathrm{dm}^{-3}\right)$ is the mole of the effective crosslinks per unit volume in the undeformed state and $v_{2}$ is the volume fraction of polymer. The bulk modulus $G$ is related to the tensile modulus $E$ as $^{21}$

$$
G=E /\left(1+2 \lambda^{-3}\right)
$$

Table II. Effective crosslink density $v_{\mathrm{e}}$ of

\begin{tabular}{|c|c|c|c|c|}
\hline \multirow{2}{*}{$\begin{array}{l}\text { Sample } \\
\text { code }\end{array}$} & \multirow{2}{*}{$v_{2} \times 10^{2}$} & \multirow{2}{*}{$\frac{\mathrm{G} \times 10^{2}}{\mathrm{MPa}}$} & \multirow{2}{*}{$\frac{v_{\mathrm{t}} \times 10^{2}}{\mathrm{moldm}^{-3}}$} & \multirow{2}{*}{$\frac{v_{\mathrm{e}} \times 10^{2}}{\mathrm{~mol} \mathrm{dm}^{-3}}$} \\
\hline & & & & \\
\hline PHEG-3 & 8.20 & 5.12 & 16.7 & 4.92 \\
\hline PHEG-4 & 10.1 & 7.10 & 22.2 & 6.38 \\
\hline PHEG-5 & 10.4 & 8.83 & 27.8 & 7.84 \\
\hline PHPG-1 & 5.04 & 3.24 & 5.55 & 3.67 \\
\hline PHPG-1.5 & 6.92 & 5.19 & 8.33 & 5.29 \\
\hline PHPG-2 & 8.17 & 6.71 & 11.1 & 6.45 \\
\hline PHPG-2.5 & 9.33 & 8.63 & 13.9 & 7.96 \\
\hline PHPG-3 & 11.0 & 10.6 & 16.7 & 9.26 \\
\hline PHPG-4 & 14.6 & 30.3 & 22.2 & 24.0 \\
\hline PHPG-5 & 25.5 & 131 & 27.8 & 82.6 \\
\hline PHBG-0.7 & 12.3 & 7.75 & 3.89 & 4.03 \\
\hline PHBG-1 & 15.2 & 9.83 & 5.55 & 5.47 \\
\hline PHBG-1.5 & 18.5 & 12.8 & 8.33 & 8.02 \\
\hline PHBG-2 & 20.7 & 15.7 & 11.1 & 10.5 \\
\hline PHBG-3 & 34.5 & 55.7 & 16.7 & 33.1 \\
\hline PHBG-4 & 40.7 & 102 & 22.2 & 57.4 \\
\hline PHBG-5 & 46.3 & 157 & 27.8 & 84.5 \\
\hline PHPeG-1 & 47.9 & 105 & 5.55 & 56.3 \\
\hline PHPeG-2 & 48.3 & 246 & 11.1 & 131 \\
\hline PHPeG-3 & 48.3 & 261 & 16.7 & 137 \\
\hline PHPeG-4 & 48.3 & 533 & 22.2 & 284 \\
\hline PHPeG-5 & 48.3 & 617 & 27.8 & 328 \\
\hline
\end{tabular}
poly $(N$-hydroxyalkyl L-glutamine) hydrogels at $298 \mathrm{~K}$

The theoretical crosslink density $v_{\mathrm{t}}$ is given by ${ }^{22}$

$$
v_{\mathrm{t}}=C f / 2
$$

where $C\left(\mathrm{~mol} \mathrm{dm}^{-3}\right)$ is the concentration of the added crosslinking agent of functionality $f$. The value of $f$ of OMDA used in this study is 4. The relation between the effective $v_{\mathrm{e}}$ with theoretical crosslink density $v_{\mathrm{t}}$ in PHG hydrogels is shown in Table II and Figure 5. Linear relationships between $v_{\mathrm{e}}$ and $v_{\mathrm{t}}$ are obtained for PHEG, PHPG and PHBG hydrogel systems. The linear dependence is given in the following equations.

$$
\begin{array}{ll}
\text { PHEG; } & v_{\mathrm{e}}=0.30 v_{\mathrm{t}} \\
\text { PHPG; } & v_{\mathrm{c}}=0.48 v_{\mathrm{t}}+0.006 \\
\text { PHBG; } & v_{\mathrm{e}}=0.63 v_{\mathrm{t}}+0.039
\end{array}
$$




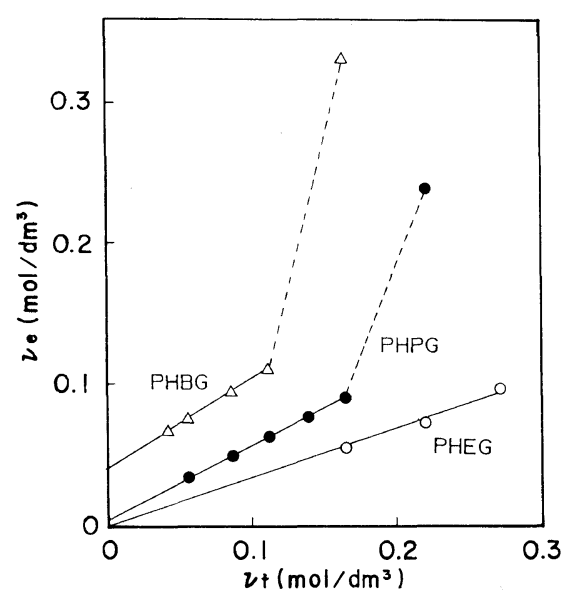

Figure 5. Relationship of effective crosslink density $v_{\mathrm{e}}$ with theoretical crosslink density $v_{\mathrm{t}}$ on $\operatorname{poly}(N$-hydroxyalkyl L-glutamine) hydrogels.

Thus, effective crosslink density was shown to be proportional to the concentration of added OMDA. The slopes in Figure 5 express the efficiency of crosslinking agent (OMDA) which competes with aminoalcohol in replacing $\gamma$ BLG residues. That is to say, the OMDA in 4-amino-1-butanol (B) will react with $\gamma$-BLG more efficiently than that in $\mathrm{P}$ or $\mathrm{E}$, possibly because the longer the alkyl side chain, the less efficient is the aminoalcohol in the reaction with the $\gamma$-BLG residues. Hence, the efficiency of crosslinking in PHPeG system is probably the highest. The fact that the slope is less than unity implies that either OMDA with a long alkyl chain has lower reactivity with $\gamma$-BLG residues than the three aminoalcohols, or all the OMDA molecules incorporated in the gel do not participate in the network structure, for example, intra-molecular crosslinking and imperfect crosslinking. Furthermore, the intercepts in Figure 5 imply the existence of effective crosslink density in the absence of crosslinking agent. Mullins ${ }^{23}$ related this to the number of entanglements. However, in this case it is more plausible to relate the values of intercepts to the helix content of hydrogel as well as to concentration of entanglements. This can be explained as PHBG and PHPG in Figure 2

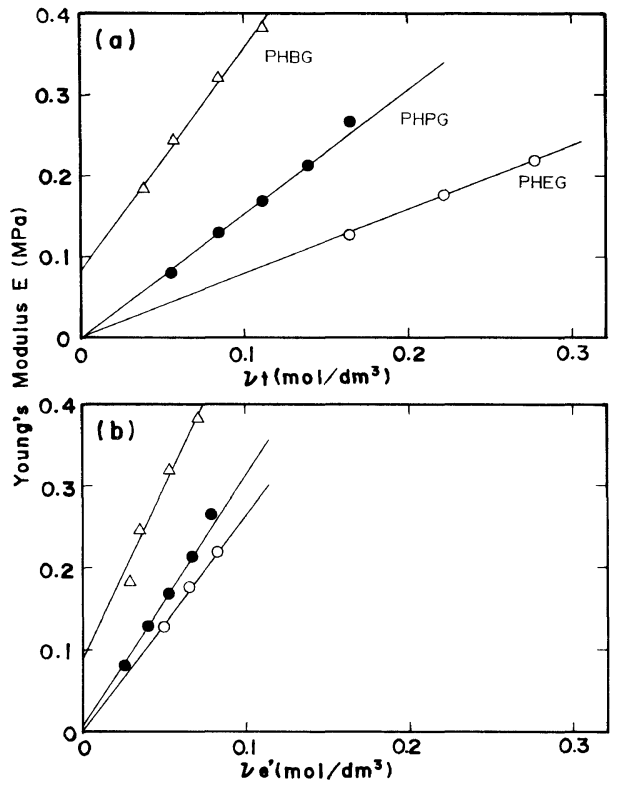

Figure 6. Effects of Young's modulus $E$ on alkyl side chain length of poly( $N$-hydroxyalkyl L-glutamine) hydrogels, PHEG ( $\bigcirc), \operatorname{PHPG}(\odot)$, and $\operatorname{PHBG}(\triangle)$, as a function of (a); concentration of crosslinking agent in the feed $v_{t}$ and (b); crosslink density in hydrogel $v_{\mathrm{e}}^{\prime}$.

show some extent of helix at 0 content of OMDA. The values of $v_{\mathrm{e}}$ in PHPG and PHBG hydrogels seem to be overestimated due to their helical structure deviating from the Gaussian chain as mentioned above. The calculated $v_{\mathrm{e}}$ of PHPG-4 and PHBG-3 deviate largely from their corresponding straight lines due to the conformational change from interrupted helix to helix.

Finally, the effect of crosslink density on Young's modulus is studied in three hydrogel systems. In Figure 6(a), Young's modulus $E$ as a function of the amount of crosslinking agent $v_{\mathrm{t}}$ is compared for three hydrogels, while in Figure 6(b) similar comparison is made for $E$ vs. $v_{\mathrm{e}}^{\prime}$ relationship where $v_{\mathrm{e}}^{\prime}$ is defined as $v_{\mathrm{e}}^{\prime}=v_{\mathrm{e}}-($ the value of the intercept $)$. The $v_{\mathrm{e}}^{\prime}$ means the crosslink density after eliminating the conformation and entanglement effects from $v_{\mathrm{e}}$. As shown in these graphs, the order of Young's modulus in these three hydrogels 
in Figure 6(a) also show up in Figure 6(b), though the difference among three hydrogels becomes smaller. It was confirmed that mechanical properties of PHG hydrogels are dependent on the alkyl side chain due to the difference in hydrophobic interaction.

Major conclusions are as follows.

(1) The effective crosslink density of poly( $N$-hydroxyalkyl L-glutamine) hydrogels can be estimated by the rubber elasticity theory.

(2) The above estimation is limited to hydrogels which contain random coil polypeptides and exhibit a skin-type behavior on the S-S curve.

(2) In comparing gels prepared under the same content of crosslinking agent in the reaction mixture, the longer the alkyl side chain of poly( $N$-hydroxyalkyl L-glutamine) hydrogel, the larger is the effective crosslink density.

\section{REFERENCES}

1. T. Sugie and P. A. Hiltner, J. Macromol. Sci-Phys., B17, 769 (1980).

2. T. Hayashi, K. Takeshima, E. Kobatake, and A Nakajima, Kobunshi Ronbunshu, 42, 777 (1985).

3. T. Hayashi, K. Takeshima, Y. Tabata, and A. Nakajima, Polym. J., 17, 1148 (1985).

4. T. Hayashi, K. Takeshima, and A. Nakajima, Polym.
J. 17, 1273 (1985).

5. P. J. Flory, "Principals of Polymer Chemistry," Cornell Univ. Press, Ithaca, N.Y., 1953, Chapter 11.

6. J. Janacek, J. Macromol. Sci., -Rev. Macromol. Chem., C9, 1 (1973).

7. I. V. Yannes, J. Macromol. Sci.-Phys., B6, 91 (1972).

8. M. B. Huglin and M. B. Zakaria, J. Appl. Polym. Sci., 31, 457 (1986).

9. L. Westman and L. Lindstrom, J. Appl. Polym. Sci., 26, 2533 (1981).

10. R. B. Woodward and C. H. Schramm, J. Am. Chem. Soc., 69, 1551 (1947).

11. T. Hayashi, K. Itoh, and A. Nakajima, Polym. J., 1, 524 (1970).

12. H. E. Auer and P. Doty, Biochemistry, 5, 1708 (1966).

13. T. Miyazawa, J. Masuda, and K. Fukushima, $J$. Polym. Sci., 62, 62 (1966).

14. N. Lotan, A. Yaron, A. Berger, and M. Sela, Biopolymers, 3, 625 (1965).

15. N. Lotan, A. S. Yaron, and A. Berger, Biopolymers, 4, 365 (1966).

16. N. Lotan, N. Bixon, and A. Berger, Biopolymers, 8 , 247 (1969).

17. P. J. Flory, "Principals of Polymer Chemistry," Cornell Univ. Press, Ithaca, N.Y., 1953, Chapter 13.

18. L. E. Neilsen, J. Macromol. Sci., C3, 69 (1969).

19. E. Nakanishi, E. Sugiyama, Y. Shimizu, S. Hibi, and T. Hayashi, Polym. J., 23, $983-990$ (1991).

20. A. V. Tobolsky, D. W. Carlson, and N. Indictor, $P$. Polym. Sci., 54, 175 (1961).

21. M. B. Huglin, M. M. A. M. Rehab, and M. B. Zakaria, Macromolcules, 19, 2986 (1986).

22. J. Janacek and J. Hasa, J. Collect. Czech. Chem. Commun., 31, 2186 (1966).

23. L. Mullins, J. Polym. Sci., 19, 225 (1956). 